\title{
Development of a bug tracking system for processing complaints to medical organizations
}

\author{
Dmitrii Demchenko ${ }^{1, *}$, Marina Bolsunovskaya ${ }^{1}$, Svetlana Shirokova ${ }^{1}$, Aleksandr \\ Leksashov $^{1}$, Ivan Orlov ${ }^{1}$, Nikita Makarevich ${ }^{1}$, and Anton Doynikov ${ }^{1}$ \\ ${ }^{1}$ Peter the Great St. Petersburg Polytechnic University, Saint-Petersburg, Russia
}

\begin{abstract}
The article substantiates the feasibility of developing and implementing an automated bug tracking system "Organization of work with citizens' appeals" to optimize the existing business processes for processing appeals and complaints to medical organizations of St. Petersburg. The article analyzes the key business processes of the enterprise in the field of registration and routing of complaints, as well as the formation of reports. The process of development and implementation of an automated system for processing requests is described, the effectiveness of implementation is assessed, and recommendations for its further development and use are given.
\end{abstract}

\section{Introduction}

Today, bug tracking systems are an integral part of the work of any IT solution development team, they help speed up the process of handling bugs in the process of creating software, as well as speed up the understanding and elimination of defects during the operation of software. This research was conducted to show that the classical logic of bug tracking systems is applicable not only in the IT field, but also in the civil sector, in such areas as education, medicine and many others. Where it is necessary to qualitatively track problems and defects during the execution of business processes.

\section{Research materials and methods}

The purpose of this work is to analyze the classic bug tracking systems market and substantiate the need to develop own solution for the St. Petersburg Medical Data Center (MDC), using the best practices from existing bug tracking systems.

The methodological basis of the study was the articles about bug-tracking systems [1-7], as well as studies on the project management systems [3,4,8,9] and software testing [8].

The object of the research is the Medical Data Center of MDC.

The main tasks of creating an automated system " Organization of work with citizens' appeals " were to ensure the possibility of accounting, systematization, and analysis of citizens 'appeals, automation of office work and document circulation by services for working with citizens' problems that arose when receiving medical care in institutions of St.

\footnotetext{
* Corresponding author: Dimademch97@gmail.com
} 
Petersburg. Allow to control the volume, as well as the timing of consideration of citizens' appeals by the responsible services, thereby improving the overall quality of medicine in the region. The main consumers of the developed solution are [2]:

- citizens of St. Petersburg, served in medical institutions of St. Petersburg;

- operators of the hotline of the health committee;

- medical staff of organizations responsible for processing requests;

- employees of the administrations of St. Petersburg, involved in the provision of medical care;

- employees of the MDC. department for work with citizens' appeals;

The bug tracking system to ensure the quality of services by medical organizations for the population of St. Petersburg, as well as to optimize the process of sending requests and their control over the fulfillment of employees' obligations [10].

\section{Results}

The paper presents a detailed description of the research object, its structure, function and business processes. The author conducted interviews of the department employees and based on it, the requirements for the necessary solution were drawn up.

After analyzing the activities of the investigated object, including the main documents, regulations and business processes [11], the need for an automated solution for high-quality business activities was identified. At the moment, most of the tasks in the department are performed manually.

During the analysis of the department's activities, the following main business processes were identified [8]:

- Processing appeals. (Registration of citizens' appeals, transmission of appeals to medical organizations and control of the received response).

- Consulting citizens by telephone hotline.

- $\quad$ Formation of reports on the work of the department.

Each process involves participants who works directly in the department for work with appeals of the MDC. (hotline, DWA operator), and external participants (MO Operators, DHC Operators, citizens-applicants, and others). Let's consider each business process separately.

Processing appeals.

Department for work with appeals process participants:

- Hotline operator - is engaged in registration appeals from citizens and transferring them to medical organizations;

- The operator for work with appeals - is engaged in monitoring the response from the medical organization.

External participants:

- Citizens of the Russian Federation wishing to leave an appeal;

- Operator of a medical organization - a person in the MO (chief physician, administrator, ...) responsible for processing citizens' appeals in his organization;

- District health department operator - a person in DHC (Polyclinics and some other medical organizations are under control of the district health department, and all legal issues must be resolved through it.), responsible for processing citizens' appeals in their district;

Description of the process, the process diagram is shown in the figure 2 [12].

Citizen's actions:

1. The citizen wants to leave an appeal for medical services that he received in a medical organization of St. Petersburg.

2. A citizen submits an appeal using one of the following options:

- Written to the mail of the department for work with appeals; 
- E-mail to the department for work with appeals;

- Oral by phone of the hotline of the department for work with appeals.

Hotline operator actions:

3. The hotline operator enters received information about the appeal into the Registration Form No. 2-OG. Information is entered in a word document. Basic information, without which the appeal is not considered: the name of the applicant, the medical organization on which the appeal is forming, gender, district and address (the street is enough) of the applicant.

4. The hotline operator checks the entered information, prints out the document and then checks the affiliation of the medical organization to DHC. For finding this information, operator uses a pdf document, where the key combination "ctrl + f' searches for a medical organization.

- If the MO belongs to DHC, the hotline operator puts the printed document in a paper folder named $\mathrm{DHC}+$ name of the $\mathrm{DHC} »$ and also saves electronic version on the server in the folder with the current day and this DHC. Further, by e-mail, the hotline operator redirects the file with an appeal to the e-mail of the district health department to which the medical organization belongs.

- If the MO does not belong to DHC, the hotline operator puts the printed document in the Health Committee folder and saves it on the server in the folder from the current day, then by e-mail it redirects the file with an appeal $t$ to the e-mail of the medical organization [13].

5. The hotline operator enters information about the registered appeal in the reports.

Actions of DHC operators:

6. Upon receipt of the complaint, the DHC Operator must arrange for a review and inform the applicant if it's necessary. And be sure to enter information about the actions taken in the registration form on the server. Informs DWA operator via e-mail.

7. If for any reason the DHC operator cannot form a response by himself, then he forwards the letter received from the hotline operator to the MO. Waits for a response from the MO operator and only after receiving an appeal and response, if the response suits him, he enters the information into the form file on the server and informs the citizen.

Actions of MO operators:

8. If the MO does not belong to the $\mathrm{DHC}$, then upon receipt of the appeal, the MO operator must arrange for a review, if necessary, inform the applicant. And be sure to enter information about the actions taken in the registration form on the server.

9. If the $\mathrm{MO}$ belongs to $\mathrm{DHC}$, then upon receipt of the appeal, the $\mathrm{MO}$ operator must arrange for a check and then send the result of the check to DHC, where the DHC operator decides whether the measures taken are sufficient and enters the information into the accounting form. Inform DHC operator via e-mail.

10. At the end of each month, the MO and DHC operators verify all accounting forms and transfer information about the processing statuses back to the MDC. to the operator for working with appeals [14]

DWA Operator Actions:

11. DWA operators check the received information, if necessary, ask the responsible $\mathrm{MO}$ and DHC operators to make corrections.

12. Print all documents and prepare the received data for analysis, enter the data processing results into intermediate tables to generate reports. 


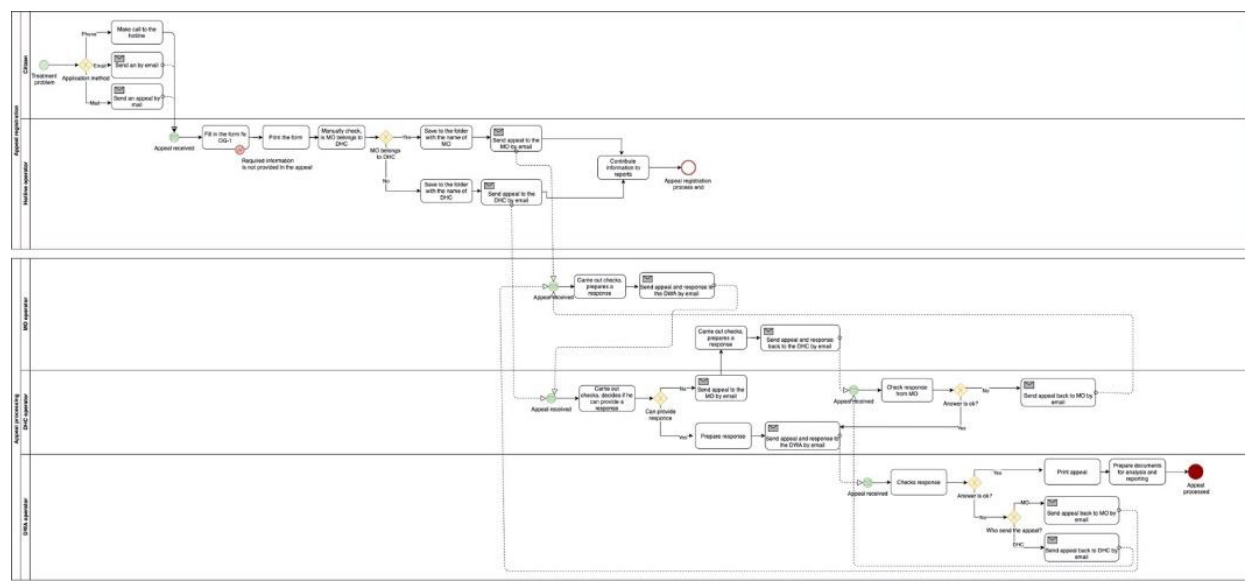

Fig. 1. AS-IS appeal processing.

Process problems:

- During the process, documentation is constantly sending via e-mail between process participants and manually uploading on server. As a result, many letters are lost and citizens cannot receive an answer to their appeals, and the problems discovered by citizens do not disappear.

- A huge amount of information is duplicated due to the need for constant re-saving and retyping of the form, the operators of $\mathrm{MO}$ and $\mathrm{DHC}$ often resubmit the forms.

- The hotline operator is forced to manually form the route of appeal, by searching the MO in the list, as a result appeals often fall to the wrong address.

- Monitoring the fulfillment of the scheduled deadlines is practically impossible, which is why most of the participants in the process are negligent in the performance of their duties

- Large costs for the salaries of operators to work with appeals, due to the need for a large staff to analyze and filter the receiving data.

Consulting citizens by telephone hotline.

Internal participants of the process:

- Hotline operator - are engaged in consulting citizens on issues that arise during treatment.

- DWA operator - collects results from all operators, generates a final report

External participants:

- Citizens wishing to use the telephone consultation

Description of the process the process diagram is shown in the figure 3 :

Citizen's actions:

1. Citizen has a need to get advice on issues about receiving medical care in institutions of St. Petersburg.

2. Citizen calls the MDC. hotline.

Hotline operator actions:

3. The hotline operator provides the necessary information to the citizen.

4. The hotline operator enters information about the type of conducted consultation in the personal report.

DWA operator

5. The DWA operator collect all data from different HL operators and make final report. 


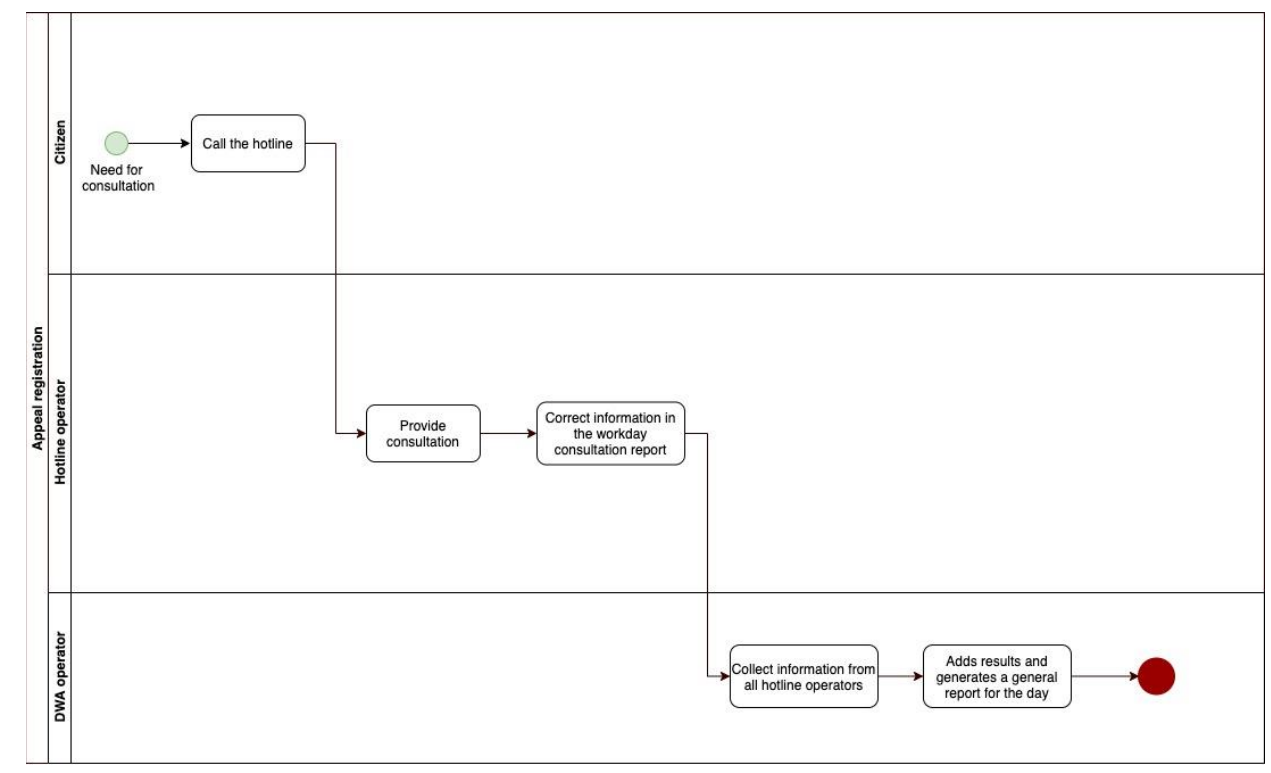

Fig. 2. AS-IS consultation processing.

Process problems:

All information is entered manually in Google docs or MS Office tables and documents, which causes errors and leads to difficulties in the analysis and processing of the obtained data. Also, it is necessary to additionally collect summary information

Formation of reports on the work of the department.

Internal participants of the process:

- Chief operator for work with appeals - distributes blocks with MO types for ordinary operators, generates a final report

- Regular operators for work with appeals - fill out report blocks

- Operators for work with appeals - are engaged in the collection and formalization of data for responsible types of medical organizations about the received appeals.

Description of the process, the process diagram is shown in the figure 4:

Actions of the main operator on handling appeals:

1. After each month, the main operator collects information from all tables with daily reports.

2. Requests information from operators who did not provide it on time.

3. Divide blocks of responsibilities (by type of medical organization: hospitals, polyclinics, children's medical institutions, counseling and private centers, maternity homes and antenatal clinics, pharmacies, sanatoriums) between other operators

Actions of other operators:

4. Operators manually enter information on their block in a special 'Excel report.

Description of the report: The first page of the report is the title page. The second page is the dynamics of appeal for the month (a histogram for comparing the total number of complaints and consultations in comparison with the same period last year). The third page is table with the structure of all appeals and consultations. Fourth - (pie chart with the ratio of all reasons for consultation). Then there are 28 pages of the report, divided into blocks of 4 pages for each type of medical organization: Information (a table with the number of complaints for each medical organization in the context of the reasons for complaints and their validity), dynamics (a histogram for comparing the number of complaints against a medical organization in comparison with a similar period last year), structure (pie chart with the ratio of causes of complaints for this type of health care), Measures taken (Table with the 
number of measures taken in the context of medical organizations of this type). Finally, at the end of the reports, there is an auxiliary page on which data is entered for the formation of graphs.

Main operator actions

5. Collects data from each operator, checks it and enters into the final report

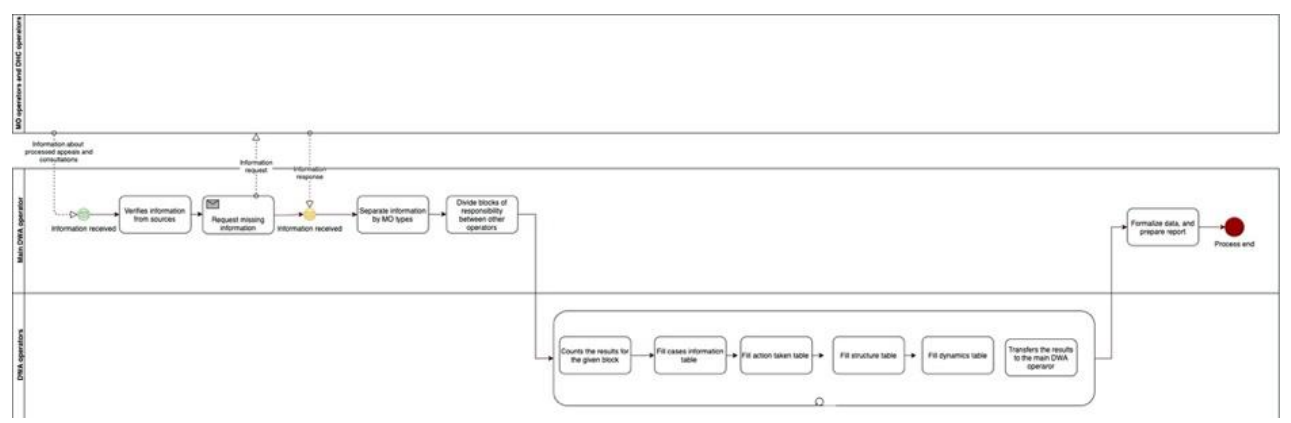

Fig. 3. AS-IS reporting process.

Process problems:

- All data for the report formation is based on daily reports, as well as on the basis of the location of the cards filled in at the stage of processing the appeal by the MO and DHC operators, which leads to losses and duplicates of information, and the quality of the information provided is low

- All pre-calculations and groupings of the received data are performed manually by the DWA operators on a calculator / Excel and other manual and semi-automated methods, that is why there are a lot of errors.

- All tabular blocks are filled in manually, while some of the information has to be duplicated in a different format to fill in the auxiliary table and create graphs.

- The time for filling out the report can be up to two weeks during periods when a large number of complaints are received and about 3-5 days with a normal load.

To solve these problems, it was proposed to use an automated bug tracking and document management system $[15,16]$. The system should monitor the effective organization of the activities of state and municipal health care institutions in St. Petersburg and ensure:

- personalized accounting of citizens' appeals, including problems that arose when receiving medical care;

- systematization and analysis of citizens' appeals;

- accounting and analysis of measures taken by the heads the medical organizations on the issues raised by the applicants;

- control of the volume, as well as the timing of consideration of appealss;

- standardization and unification methods for collecting and processing information received from citizens;

- monitoring of citizens' satisfaction with the activities of medical institutions $[17,18]$.

General requirements for an appeal processing system

Since in the process of processing applications, data is exchanged between users with different access levels and areas of responsibility in the system, a clear role model should be provided. The role model should provide for 4 main roles - the main participants in the appeal processing: Hotline Operator, MO Operator, DHC Operator, DWA Operator [19-21]. And also three additional ones: Administrator (responsible for managing the system, the bug tracking system should give the administrator the ability to configure access rights to appeals, that is, which users can view and edit appeals depending on their state, as well as transfer them to another state or delete them [24]), Controlling user (user responsible for the 
compliance of regulations - prosecutor's office, health committee), User of third-party systems (user leaving an appeal on the portal).

To determine the necessary functions, participants in the existing process were interviewed. The based received information, Use Case diagrams were drawn up for each of the roles. Use cases give a structured way of capturing the behavioral requirements of a system, so that you can reasonably create a design from them. They help you to answer some fundamental questions: What are the users of the system trying to do? What's the user experience? [22] Use Case - is a scenario technique for describing an interaction. A use case can both describe a user requirement and a description of people and companies in real life. $[23,24]$ The figure 5 shows the Use Cases for the main roles. The figure 6 shows the Use Cases for the additional roles.

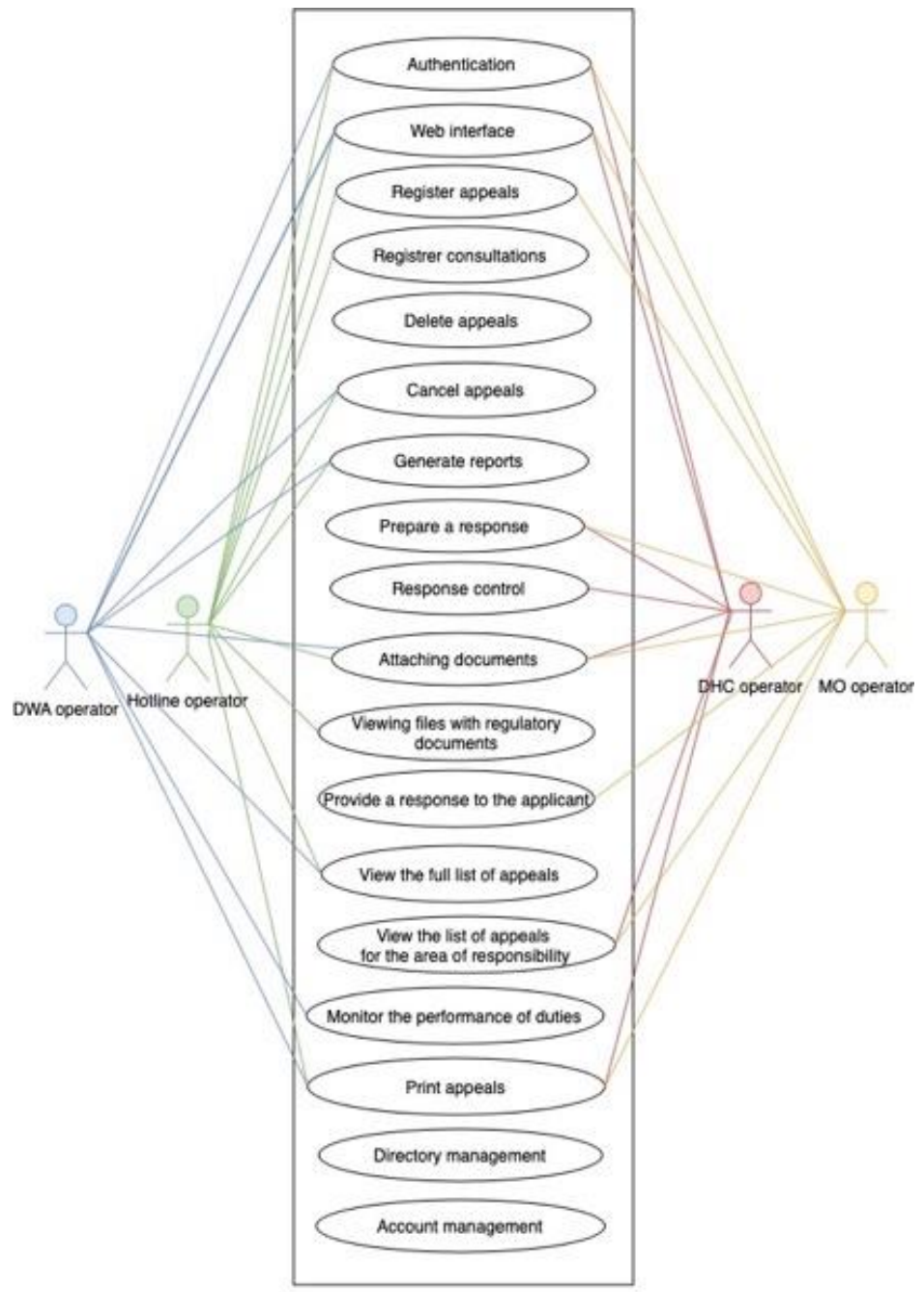

Fig. 4. AS-IS Use Case of the necessary functions for the main roles. 


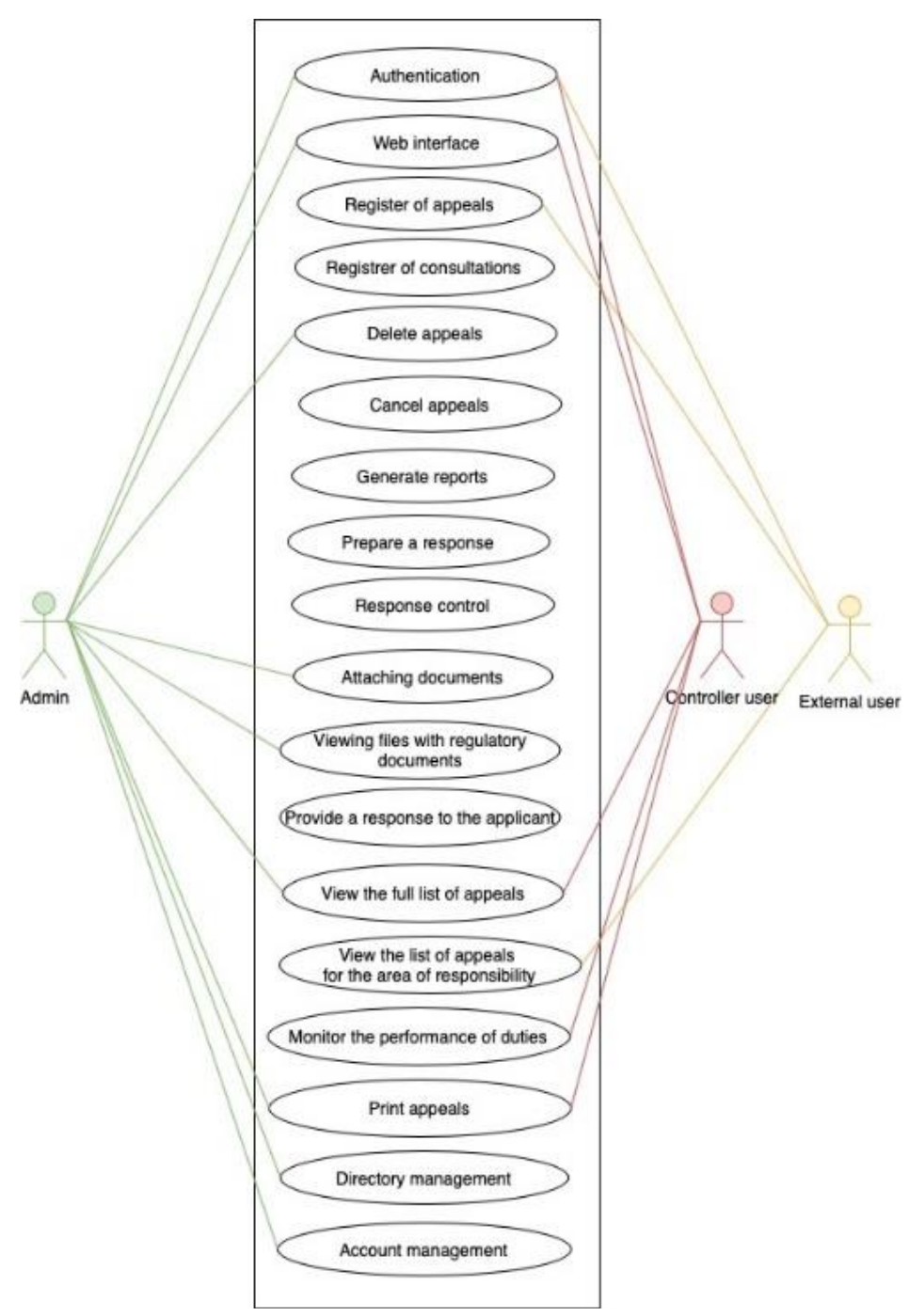

Fig. 5. AS-IS Use Case of required functions for additional roles.

During the analysis of use cases, as well as collecting requirements from stakeholders, a list of the functionality required in the system was compiled:

- Users must be able to enter the system using the login + password combination stored in the system database.

- $\quad$ All users, except for a user of a third-party system, must have access to a web interface with elements that depend on the user's role.

- $\quad$ The system administrator must be able to create / edit users. In addition, rights must be added for each function in the system in order to be able to enable additional rights to the particular role.

- Managing directories - the system administrator should be able to add / edit medical organization, DHC, reasons for contacting and consultations directories in the GUI of the system. In addition, it should be possible to upload the MO directory from the resources of the Netrik terminalology service.

- When registering an appeal in the system, it should be possible to use the address reference of SPB and LO for the correct entry of the address in accordance with legal norms. 
- The system should have clearly regulated routes along which the appeal follows, depending on the type and source.

- In some cases, under one legal entity. Several MOs can work as a one legal entity, in such cases one operator responds on appeals to all controlled MOs. It is necessary to develop a mechanism in which the operator sees which particular MO the citizen is complaining about. Since at the moment the hotline operators do not always know about the existence of subordinate organizations.

- In the system, the user should have access to appeals that are in his area of responsibility.

- The system should have able to create an appeal in accordance with the form No. 2OG.

- The system must have functionality that allows you to attach files to appeals and responses.

- The system should be able to quickly find and print the required appeal in the format of the form No. 2-OG.

- The system should be able to register the facts of consultations for the subsequent preparation of a report.

- The system should provide mechanisms for processing the appeal and preparing a response, in which the appeal can be redirected many times to the previous stages of processing. In this case, all interactions should be recorded in the comments.

- The system should provide for the possibility of using a qualified electronic signature based on Crypto PRO CSP 4.0 format.

- The system must have a mechanism for notifying the participants in the process about new appeals and coming to the end of the processing time.

- The system should have a mechanism for monitoring the work and implementation of the operator's regulations

- The system should be able to use the embedding of the interface into other systems of the information and analytical center.

- The system should have mechanisms for integration with other systems, in particular with the Internet portal "Health of St. Petersburg", while all appeals coming from third-party systems must have a route different from the standard ones (without the participation of DHC and DWA operators)

After implementing the system to meet these requirements, the following advantages were obtained in the processing of requests: consultations.

Improving the convenience of operators involved in the management of appeals and

- Increasing awareness of department for work with appeals about delays and poorquality work of MO and DHC staff.

- Increasing the satisfaction of citizens with the answers provided to them and the measures taken.

- $\quad$ Citizens receive a new way to leave an appeal (Internet portal).

- Multiple reduction in errors and duplicates due to the absence of the need to make manual calculations and mailings.

- Improving the quality of reporting, as a result of improving the quality of analytics and medicine in general, due to a more effective impact on the problems of citizens;

- $\quad$ Reducing the time spent on creating and transferring to the gate.

- $\quad$ Reducing the cost of the department for work with appeals, due to the lack of the need to constantly increase the staff due to lack of resources for reporting and monitoring obligations. 


\section{Discussion}

Within the framework of such a project, many tasks are devoted to collecting and managing requirements. Therefore, I would like to note the importance of high-quality claims management. Requirements management is a complex process that includes the development of documentation such as Vision and Scope, SRS, Use Cases, Sustainability Analysis. Additional documents [25]. And also to say that when managing such projects and simplifying the work with requirements management processes, it is possible to use specialized systems that allow, when creating a large amount of documentation, to determine the requirements and establish a trace between them, it becomes possible to track changes in these parts. Thus, if any part of one of the documents has been changed, then it is easy to track which parts of this or another document need to be viewed first to make possible corrections to them [26].

\section{Conclusion}

Thus, in accordance with the described business processes, the following are implemented in IS "OWSA":

- automation of document flow between participants in the process of consideration of the application;

- automation of control and analysis of the results of the activities carried out in MOs based on the results of consideration of appeals;

- automation of planning, management and evaluation of the activities of services dealing with citizens' appeals;

- analytical processing of poorly ordered information based on the results of citizens' appeals in order to bring it to a standardized and / or unified form

At the moment, the department for work with appeals has been using the system for a year, thanks to which the time spent by employees has decreased by $40 \%$. The implementation of the system is in full swing in all medical organizations of St. Petersburg. MDC entered contracts with the developer of contracts for the development of additional functionality in the context of new reports and integrations with other government systems. Moreover, during the analysis of economic efficiency, it was revealed that even though the project is mainly created to improve quality indicators and payback in government projects is not always a priority. The project will fully pay off by 3 years of operation.

\section{References}

1. N.A. Alekseev, A. Galunina, K.S. Polyachka, A.A. Sbitneva, Education and science in Russia and abroad 2, 37 - 41 (2019)

2. MIAC, https://spbmiac.ru

3. N.V. Ivavanov, Forum of young scientists. Institute of Management and SocioEconomic Development "(Saratov) 7, 290 - 298 (2017)

4. A.G. Madera, Business processes and process management in conditions of uncertainty: Quantitative modeling and optimization (Lenand, M., 2019)

5. A. Sokolova, Modern information technology and it education 2, 146 - 149 (2015)

6. S. Just, A. Schroter, C. Weiss, R. Premraj, T. Zimmermann, Proceedings of the 16th International Symposium on Foundations of Software Engineering, 308 -318 (2008)

7. J. Aranda, G. Venolia, Proceedings of the 31st International Conference on Software Engineering, 11-13 (2009) 
8. S. Kulikov, Software testing (EPAM Systems, 2015)

9. PM1. Project Management Guide. Body of knowledge: RMVOK Oshbe. Fifth edition (Project Management Institute, 2013)

10. OOS. Management of successful projects with RC1KSE2 (TZO, London, 2009)

11. Bugzilla, https://www.bugzilla.org

12. I.V. Ilyin, O.U. Ilyashenko, Science and business: ways of development, 17- 24 (2016)

13. M. Uspenskij, A. Makarov, A. Sochnev et al., Proceedings of the 33rd International Business Information Management Association Conference, IBIMA 2019: Education Excellence and Innovation Management through Vision 2020, 8508-8514 (2019)

14. S.V. Krasnov, S.A. Krasnova, Problems of Economics and Management in Trade and Industry 4(4), 21-25 (2013)

15. A. Edward, J. Wesley, Workflow Automation: Overview and Research Issues (Howe School of Technology Management,2001)

16. S. Breu, R. Premraj, T. Zimmermann, J Frequently asked questions in bug reports (University of Calgary, 2009)

17. O. Rostova, A. Zabolotneva, A. Dubgorn et al., Proceedings of the 33rd International Business Information Management Association Conference, IBIMA 2019: Education Excellence and Innovation Management through Vision 2020, 8525-8530 (2019)

18. P. Hooimeijer, W. Weimer, ASE'07: Proceedings of the 22nd International Conference on Automated Software Engineering, 34-43 (2007)

19. Jira software, https://ru.atlassian.com/software/jira/bug-tracking

20. Redmine, https://www.redmine.org

21. Laws, codes and regulatory legal acts of the Russian Federation, https://legalacts.ru

22. D. Rosenberg, M. Stephens, Apress, 49-50 (2007)

23. What is use case and why are they needed, https://systems.education/use-case

24. A.A. Lepekhin, V.M. Ilyashenko, Collection: SPbPU Science Week. Materials of a scientific conference with international participation (SPb, 2017)

25. I. Garcia, IEEE Latin America Transactions, 4181 - 4186 (2012)

26. J.C. Helm, I. Bozkurt, Technology Interface International Journal, 39-48 (2013) 